\title{
When is Lugol still necessary in 2020?
}

\section{(ㄷ) (1) $(2) \div$}

\author{
Authors \\ Steffi E.M. van de Ven, Arjun D. Koch \\ Institution \\ Department of Gastroenterology and Hepatology, Erasmus \\ MC, University Medical Center, Rotterdam, The Netherlands \\ Bibliography \\ Endoscopy International Open 2020; 08: E1478-E1480 \\ DOI 10.1055/a-1216-1864 \\ ISSN 2364-3722 \\ (c) 2020. The Author(s).
}

\begin{abstract}
This is an open access article published by Thieme under the terms of the Creative Commons Attribution-NonDerivative-NonCommercial License, permitting copying and reproduction so long as the original work is given appropriate credit. Contents may not be used for commecial purposes, or adapted, remixed, transformed or built upon. (https://creativecommons.org/licenses/by-nc-nd/4.0/)
\end{abstract}

Corresponding author

Arjun D. Koch, Postbox 2040, 3000 CA Rotterdam, the Netherlands

Phone: +316 24463113

a.d.koch@erasmusmc.nl
Detection of early esophageal squamous cell carcinoma (ESCC) is very important, because these cancers can be treated with minimally invasive endoscopic resection instead of surgery. Early ESCC is characterized by subtle flat lesions, which are easily overlooked during routine white light endoscopy [1]. To improve ESCC detection, the addition of Lugol iodine was introduced [2]. Today, Lugol dye chromoendoscopy (LCE) is considered by many the gold standard for detection of early ESCC [3, 4].

Lugol iodine was first used in the esophagus to screen for ESCC in 1966 [2]. In the esophagus, Lugol iodine binds to glycogen [5]. Glycogen is diminished or absent in dysplastic or neoplastic tissue and abundant in normal squamous epithelium [5]. As a result, areas with dysplasia or neoplasia have reduced or even absent iodine staining, whereas normal squamous epithelium is intensely stained by Lugol iodine [5]. Although these unstained areas, so-called Lugol voiding lesions, make it more easy to detect dysplasia or neoplasia, non-dysplastic lesions such as inflammation can also appear unstained [1]. As a consequence, LCE is highly sensitive but not very specific in the detection of ESCC [1].

In addition, several side effects of LCE have been described, such as chest discomfort, heartburn, nausea, pulmonary aspiration, and allergic reaction [6,7]. Another disadvantage for both the patient and endoscopist is the extended procedure time [7]. Different concentrations of iodine solution ( $1 \%$ to $3 \%$ ) have been used in studies, and patient discomfort seems to depend on the iodine concentration used $[8,9]$. A recent randomized controlled trial showed that use of $1 \%$ iodine solution resulted in less heartburn and retrosternal pain compared to $2 \%$ iodine solution $(P=0.02)$ [8]. In both groups, the color of the stained esophageal images was similar [8]. LCE with $1 \%$ iodine solution, therefore, is recommended [8].
Early ESCC can also be identified by narrow-band imaging (NBI) as brown, well-demarcated lesions [6]. This is a real-time optical chromoendoscopy technique that was first described in 2004 [10]. It visualizes the mucosa and intraepithelial papillary capillary loop (IPCL) patterns [11]. Although NBI is easy to use by pressing a button on the endoscope, the device is expensive and expertise is required [6]. For example, for inexperienced endoscopists, it might be difficult to distinguish inflammation from dysplastic lesions using NBI.

Because endoscopic imaging techniques have drastically improved over time and Lugol has several side effects, the questions arises whether and when Lugol is still necessary.

Before we completely abandon use of Lugol based on current knowledge and the evidence presented by Costa-Santos et al., we have to consider different phases in the endoscopic treatment of ESSC. There are three important phases that finally lead to endoscopic treatment. First, lesions have to be detected. A second important step is characterization and if deemed amendable for endoscopic resection, the final important step is delineation. All three steps can be done using either Lugol or NBI. The question arises whether NBI is superior to Lu$\mathrm{gol}$ in all three steps or there are distinct advantages for each technique in the separate steps.

\section{Lesion detection}

Several studies have compared accuracy of LCE with NBI in detection of early ESCC. Wang et al. reported that the combination of NBI and LCE in detection of ESCC showed the highest sensitivity (94.7\%), compared to LCE (93.0\%) and NBI (84.2\%) alone [9]. A recent systematic review and meta-analysis, including 12 studies, showed that the sensitivity of NBI and LCE were comparable ( $88 \%$ vs. $92 \%$ ) and the specificity was superior with 
$\mathrm{NBI}$ ( $88 \%$ vs. $82 \%, P<0.001)$ [12]. However, all endoscopies were performed by expert endoscopists $[9,12]$. Therefore, accuracy rates may be lower when performed by a general endoscopist.

\section{Lesion characterization}

Esophageal lesions suspected of being dysplastic or neoplastic are highlighted by LCE as Lugol voiding lesions. Lugol voiding lesions are present or not, but no further characterization of these lesions can be made besides gross morphology. As a consequence, it might be difficult to distinguish inflammation from dysplasia or neoplasia or deeper invasion outside criteria for endoscopic treatment. In contrast to LCE, IPCLs visible with $\mathrm{NBI}$ can further characterize esophageal lesions. For example, variety in IPCL shapes, tortuous IPCL, presence of a demarcation line, lesions with brownish dots or brownish epithelium all were associated with mucosal high-grade neoplasia, according to Ishihara et al [13]. Based on presence of brownish dots or brownish epithelium, the sensitivity for detecting neoplasia was $100 \%$ [13].

\section{Lesion delineation}

In the current issue of this journal, Costa-Santos et al. compared the effectiveness of NBI and LCE in defining lateral resection margins before endoscopic resection of ESCC and dysplasia [14]. Studies on this important step in endoscopic treatment have not been previously reported. In their study, two groups of patients with ESCC or dysplasia who underwent en-bloc resection were defined: (1) inspection with NBI only; and (2) inspection with LCE (with or without NBI). Of 132 included lesions, 68 (52\%) were inspected with LCE and 64 (48\%) with $\mathrm{NBI}$ only. The complete lateral resection rate for invasive carcinoma did not differ between the two groups; the resection rate was $90 \%$ in the LCE group and $94 \%$ in the NBI group ( $P=0.715)$. Also, the lateral resection rate for dysplasia did not differ between the LCE $(65 \%)$ and NBI $(67 \%)$ groups ( $P=0.813)$. CostaSantos et al. concluded that mucosal inspection with LCE before endoscopic resection of ESCC and dysplasia was not associated with an increased complete lateral resection rate compared to inspection with $\mathrm{NBI}$ alone [14].

The results of this study are very interesting and add to our knowledge about minimal invasive endoscopic treatment of early cancer. This study supports use of NBI for delineation but it does not show superiority of NBI versus Lugol. NBI was used in a recent cohort in which modern endoscopes were used, in contrast to the historical LCE cohort in which older endoscopes were used. NBI was combined with a white light imaging technique that has dramatically improved over the years, with improved magnification and image resolution. Results with the combination cannot be separated from use of NBI "alone." In contrast, older endoscopes used in the LCE cohort did not display these superior features. Therefore, the technology in these two different cohorts with different endoscopes might have influenced the results of Costa-Santos et al. A fair comparison would be using both techniques with the same superior endo- scopes. In addition, the authors did not clearly report on how many lesions in the LCE group were also inspected with NBI. Inspection with NBI before Lugol iodine staining may influence on the definition of lateral resection margins before endoscopic resection.

For both NBI and LCE, adequate expertise and experience on the part of the endoscopist is key in detection, characterization, and delineation of esophageal lesions. Although NBI seems superior in terms of specificity and characterization of lesions, detection of lesions depends on the endoscopist's experience. Recognition of specific IPCL patterns is crucial in NBI, while detection of Lugol voiding lesion by LCE might be easier for an endoscopist with less experience. Future developments might very well include artificial intelligence. Computer algorithms can "red flag" or even characterize suspicious areas. They are already part of video capsule endoscopy and their use is increasing in colonoscopy screening $[15,16]$. In all likelihood, computer algorithms will make use of these superior imaging techniques and the role of Lugol will be pushed more to the background. Until that new era arrives, Lugol can still be a very useful "red flag" in detection of early squamous neoplasia.

\section{Competing interests}

The authors declare that they have no conflict of interest.

\section{References}

[1] Goda K, Dobashi A, Yoshimura N et al. Narrow-Band imaging magnifying endoscopy versus lugol chromoendoscopy with pink-color sign assessment in the diagnosis of superficial esophageal squamous neoplasms: a randomised noninferiority trial. Gastroenterol Res Pract 2015; 2015: 639462

[2] Voegeli R. Schiller's iodine test in the diagnosis of esophageal diseases. Preliminary report. Pract Otorhinolaryngol (Basel) 1966; 28: 230-239

[3] Bugter O, van de Ven SEM, Hardillo JA et al. Early detection of esophageal second primary tumors using Lugol chromoendoscopy in patients with head and neck cancer: A systematic review and meta-analysis. Head Neck 2019; 41: 1122-1130

[4] Inoue H, Rey JF, Lightdale C. Lugol chromoendoscopy for esophageal squamous cell cancer. Endoscopy 2001; 33: 75-79

[5] Mori M, Adachi Y, Matsushima T et al. Lugol staining pattern and histology of esophageal lesions. Am J Gastroenterol 1993; 88: 701-705

[6] Lopes AB, Fagundes RB. Esophageal squamous cell carcinoma - precursor lesions and early diagnosis. World J Gastrointest Endosc 2012; 4: 9-16

[7] Dubuc J, Legoux J-L, Winnock M et al. Endoscopic screening for esophageal squamous-cell carcinoma in high-risk patients: a prospective study conducted in 62 french endoscopy centers. Endoscopy 2006; 38: 690-695

[8] Gotoda T, Kanzaki H, Okamoto Y et al. Tolerability and efficacy of the concentration of iodine solution during esophageal chromoendoscopy: a double-blind randomized controlled trial. Gastrointest Endosc 2020; 91: 763-770

[9] Wang CH, Lee YC, Wang CP et al. Use of transnasal endoscopy for screening of esophageal squamous cell carcinoma in high-risk pa- 
tients: yield rate, completion rate, and safety. Digest Endosc 2014; 26: $24-31$

[10] Gono K, Obi T, Yamaguchi M et al. Appearance of enhanced tissue features in narrow-band endoscopic imaging. J Biomed Opt 2004; 9: 568-577

[11] Yoshida T, Inoue H, Usui S et al. Narrow-band imaging system with magnifying endoscopy for superficial esophageal lesions. Gastrointest Endosc 2004; 59: 288-295

[12] Morita FH, Bernardo WM, Ide E et al. Narrow band imaging versus lugol chromoendoscopy to diagnose squamous cell carcinoma of the esophagus: a systematic review and meta-analysis. BMC Cancer 2017; 17: 54

[13] Ishihara R, Inoue T, Uedo $\mathrm{N}$ et al. Significance of each narrow-band imaging finding in diagnosing squamous mucosal high-grade neo- plasia of the esophagus. J Gastroenterol Hepatol 2010; 25: 1410 1415

[14] Costa-Santos Maria Pia, Ferreira Alexandre Oliveira, Mouradides Christina et al. Is Lugol necessary for endoscopic resection of esophageal squamous cell neoplasia? Endosc Int Open 2020; 08: E1471E1477

[15] Ahmad OF, Soares AS, Mazomenos E et al. Artificial intelligence and computer-aided diagnosis in colonoscopy: current evidence and future directions. Lancet Gastroenterol Hepatol 2019; 4: 71-80

[16] Ding Z, Shi H, Zhang H et al. Gastroenterologist-level identification of small-bowel diseases and normal variants by capsule endoscopy using a deep-learning model. Gastroenterology 2019; 157: 1044-1054 e1045 